\title{
Agroindustrial Science
}

Escuela de Ingeniería

Agroindustrial

Website: $\underline{\text { http://revistas.unitru.edu.pe/index.php/agroindscience }}$

Universidad Nacional de Trujillo

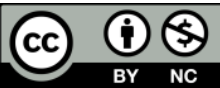

Esta obra está publicada bajo la licencia CC BY-NC 4.0

\section{Percepción sensorial de un análogo de carne (tofu) mediante métodos descriptivos rápidos}

\author{
Sensory perception of a meat analog (tofu) using rapid descriptive methods
}

\section{Olivia Diana Morales Bustos"; Amparo Eccoña Sota1; Reynaldo Justino Silva Paz ${ }^{1, \text { * }}$}

\author{
1 Escuela de Ingeniería de Industrias Alimentarias, Facultad de Ingeniería y Arquitectura, Universidad Peruana Unión, Carretera \\ Central Km 19.5 Ñaña, Chosica, Lima, Perú.
}

ORCID de los autores:

O. D. Morales Bustos: https://orcid.org/0000-0002-2128-1628

A. Eccoña Sota: https://orcid.org/0000-0001-9418-2754

R. J. Silva Paz: https://orcid.org/0000-0003-4400-7469

\section{RESUMEN}

Los métodos descriptivos ayudan a definir las características perceptibles de diversos productos. La presente investigación tuvo como objetivo describir sensorialmente mediante dos métodos descriptivos rápidos un análogo de carne (Tofu) elaborado con tarwi (Lupinus mutabilis). Para ello se aplicando el perfil de libre elección y la metodología CATA. Se trabajó con ocho muestras variando el porcentaje de soya, tarwi y lactato de calcio. Para el perfil de libre elección se realizó un Análisis Procrustes Generalizado y para CATA se aplicó la prueba $Q$ Cochran y análisis de correspondencia. Los datos fueron analizados con el programa estadístico R v.3.5.3. El perfil libre describió a las muestras como suaves, porosas, adhesivas, pastosas, terrosas, elásticas, olor a soya, arenosas, sabor a soya y color amarillo, donde se observó la formación de cuatro grupos (dos muestras por grupo). El método CATA generó dos grupos, el primer grupo conformado por la muestra A y B, descriptas como pastosas, color amarillo, adhesivas, elásticas, fundentes, suaves; y el segundo grupo formado por el resto de las muestras caracterizadas por olor a soya, sabor y olor a leche, esponjoso, chicloso y blanco. Ambas metodologías mostraron resultados similares en la descripción de las muestras de análogo de carne.

Palabras clave: Tofu; análogo de carne; características sensoriales; perfil libre; CATA.

\section{ABSTRACT}

Descriptive methods help define the perceptible characteristics of various products. The objective of the present investigation was to describe a meat analogue (Tofu) made with tarwi (Lupinus mutabilis) by means of two rapid descriptive methods. For this, the free-choice profile and the CATA methodology are applied. Eight samples were used, varying the percentage of soy, tarwi and calcium lactate. For the free-choice profile, a Generalized Procrustes Analysis was performed and for CATA, the $\mathrm{Q}$ Cochran test and correspondence analysis were applied. The data were analyzed with the statistical program R v.3.5.3. The free profile described the samples as soft, porous, sticky, pasty, earthy, elastic, soy odor, sandy, soy flavor and yellow color, where the formation of four groups (two samples per group) was observed. The CATA method generated two groups, the first group made up of samples A and B, described as pasty, yellow, adhesive, elastic, flux, soft; and the second group formed by the rest of the samples characterized by the smell of soy, the taste and smell of milk, fluffy, chewy and white. Both methodologies showed similar results in the description of the meat analog samples.

Keywords: Tofu; meat analogue; sensory characteristics; Free Choice Profile; CATA. 


\section{Introducción}

El tofu es un alimento de origen vegetal muy nutritivo con un aumento demanda mundial (Nikolić et al., 2017). Los productos como el tofu y los derivados de la soya se consumen cada vez más, esto debido al incremento de la concientización de la alimentación saludable. Sin embargo, los principales atributos de calidad que caracterizan al tofu son las fisicoquímicas y sensoriales que caracterizan al tofu (Zhang \& Qin, 2019).

Durante la fabricación, el tofu es preparado con la leche de soya caliente con coagulantes, seguido de un moldeado y prensado de la parte coagulada para eliminar el suero (Murugkar, 2015). La soya, el ingrediente principal del tofu, es uno de los más cultivados cultivos a nivel mundial (Pizzutti et al., 2007). Por su parte, el tarwi (Lupinus mutabilis) tiene muy buenas propiedades nutricionales, con uno de los niveles más altos de proteínas entre las leguminosas y un excelente perfil de aminoácidos. Además, contiene un alto nivel de carbohidratos y fibra no digestibles (Van de Noort, 2017).

La caracterización sensorial es una de las herramientas más poderosas, sofisticadas y ampliamente aplicada en la ciencia sensorial, ya que proporciona una representación de los aspectos cualitativos y cuantitativos de la percepción humana. Esto permite medir la reacción sensorial a los estímulos generados por un producto (Kharlamova et al., 2018).

Los análisis descriptivos se emplean continuamente con el objetivo de capturar la intensidad de las propiedades sensoriales que difieren entre un grupo de muestras (Pearson et al., 2020). El perfil de libre elección permite a los consumidores describir y evaluar productos en su propia terminología (Elmore \& Heymann, 1999). Este perfil no requiere una alineación construida, pero los evaluadores deben ser consistente en el uso del descriptor (Narain et al., 2004).

La evaluación sensorial por parte de consumidores y jueces no capacitados se ha convertido en un tema de investigación para la ciencia sensorial. Existe una creciente evidencia científica de que los jueces sin capacitación pueden usarse para describir las características generales de un producto en particular. Este hecho proporciona una solución al problema presentado por las industrias alimentarias y puede usarse como una herramienta para el desarrollo y caracterización de nuevos productos (Zhang \& Qin, 2019).

En los últimos años ha aumentado el interés por los métodos sensoriales que son más rápidos y fáciles de usar (Pearson et al., 2020). estos han sido aplicados en mandarinas (Tarancón et al., 2020), vinos (Pelonnier-Magimel et al., 2020), jugos de manzana (Nascimento et al., 2020), como en productos no alimentarios: raquetas de tenis (Bauer et al., 2020). Por tal razón, la presente investigación tuvo como objetivo describir sensorialmente mediante dos métodos descriptivos rápidos la sustitución del tarwi (Lupinus mutabilis) en la elaboración de tofu.

\section{Material y métodos}

\section{Materia prima e insumos}

Los granos de soya (Glycine max) y tarwi (Lupinus mutabilis) fueron obtenidos del mercado central de Lima y el lactato de calcio marca MERRYYANG, fue adquirido en la empresa Polifood Perú S.A.C.

\section{Proceso de formulación y elaboración del tofu} Se desarrollaron 8 muestras de tofu, cada formulación se modificó el porcentaje de las materias primas y el coagulante, tal como se muestra en la Tabla 1. Para la preparación de tofu se siguió la metodología de Meng et al. (2016), los granos de soya se sometieron a un enjuague previo y se pasó a un remojo en agua a temperatura ambiente por un periodo de 10 horas, pasado este tiempo se eliminó el agua que no fue absorbida por los granos y se mezcló con nueva agua, la relación de semillas de soya y agua fue 1:6 para pasar al proceso de molienda y obtener la leche de soya. En el caso del tarwi, después de haber pasado por un proceso de desamargado, los granos se encontraban listos para pasar al proceso de pesado, en este paso, los granos de tarwi como de soya se mezclan para luego pasar al proceso de molienda y obtener la leche. La leche se filtró en telas de poliseda. Después del filtrado, se calentó hasta llegar al punto de ebullición y se mantuvo durante $10 \mathrm{~min}$. Después que la leche se enfría y llega a una temperatura de $85^{\circ} \mathrm{C}$ se agregó el coagulante (lactato de calcio) y se dejó coagular por un periodo de 45 min. Finalmente, se llevó a realizar el prensado con el objetivo de eliminar la parte acuosa de la sólida (desuerado) y se almacenó a $4{ }^{\circ} \mathrm{C}$ (Li et al., 2017).

\section{Perfil de libre elección}

El perfil de libre elección se llevó a cabo con 9 consumidores en tres sesiones, con una duración promedio de $45 \mathrm{~min}$ en total. La primera sesión fue una descripción de la metodología, y la generación del vocabulario propio de los consumidores. La segunda fue la consolidación de los descriptores y una prueba preliminar para 
que los panelistas se adapten al uso de la escala. Finalmente, la tercera sesión fue la descripción sensorial de las muestras mediante la escala de intensidad. Para evaluar la intensidad de los atributos se utilizó una escala no estructurada de $10 \mathrm{~cm}$ (muy baja intensidad - muy alta intensidad) (Liu et al., 2018).

\section{Evaluación de Perfil CATA}

Se aplicó la prueba CATA ("Marque todo lo que corresponda") de las 8 muestras de Tofu a 53 consumidores, con edades entre 20 a 50 años. La prueba presentó 23 atributos (generados previamente por los panelistas durante una de las sesiones del perfil de libre elección). Se indicó a los panelistas que verificaran cada atributo que consideraban apropiado. Las muestras se codificaron con tres dígitos aleatorios diferentes y se presentaron de forma monádica aleatorizada (Esmerino et al., 2017).

Tabla 1

Formulación de las muestras de tofu

\begin{tabular}{cccc}
\hline Muestra & $\begin{array}{c}\text { Soya } \\
(\%)\end{array}$ & $\begin{array}{c}\text { Tarwi } \\
(\%)\end{array}$ & $\begin{array}{c}\text { Lactato de calcio } \\
(\%)\end{array}$ \\
\hline A & 0 & 100 & 0,3 \\
B & 0 & 100 & 0,5 \\
C & 40 & 60 & 0,3 \\
D & 40 & 60 & 0,5 \\
E & 80 & 20 & 0,3 \\
F & 80 & 20 & 0,5 \\
G & 100 & 0 & 0,3 \\
H & 100 & 0 & 0,5 \\
\hline
\end{tabular}

\section{Análisis Estadístico}

Los resultados del perfil de libre elección se analizaron mediante Análisis Procrustes Generalizado (GPA), donde cada consumidor genera una tabla de datos independiente, y el análisis busca una estructura común entre las diferentes tablas mediante la normalización de los resultados al dividir cada tabla entre el primer autovalor generado en un análisis de componentes principales aplicados previamente a cada tabla individual. El GPA proporciona por tanto un consenso entre el análisis de los individuos entre las diferentes tablas o resultados de los jueces. Además, se determinó el coeficiente Rc.

Para el método CATA, los atributos se evaluaron mediante la prueba Q-Cochran para identificar los atributos que tuvieron diferencias significativas en las proporciones de marcado. En base a los atributos significativos se construyó una tabla de contingencia y se evaluó la relación global entre muestra, los resultados fueron visualizados según el método de correspondencias simple (CA). Los datos fueron analizados en el programa $R$ v 3.5.3 utilizando la librería FactoMineR (Esmerino et al., 2017).

\section{Resultados y discusión}

\section{Perfil de libre elección}

Análisis Procrustes Generalizado aplicado al Perfil de Libre elección

Los resultados del análisis de varianza de Procrustes Generalizado (PANOVA), indicaron que la fuente de traslación $(\mathrm{F}=4,903$ y $\mathrm{Pr}>\mathrm{F}=$ $<0,0001)$ y escalamiento $(F=2,046$ y $\mathrm{Pr}>\mathrm{F}=$ 0,044 ) tienen un efecto significativo en la reducción de la variabilidad de las configuraciones, mientras que la fuente de rotación $(F=1,142$ y $\mathrm{Pr}>\mathrm{F}=0,158)$ no presenta un efecto significativo en la contribución de permutación sobre la variabilidad de las configuraciones.

Para realizar el perfil de libre elección, se contó con 9 consumidores entre 18 y 25 años. Este número de evaluadores es similar a los realizados por Vit et al. (2017) donde participaron 8 evaluadores para realizar la descripción de diferentes tipos de mieles.

\section{Prueba de consenso de los consumidores}

El coeficiente de correlación (Rc) para los consumidores fue de $\mathrm{Rc}=0,60(60 \%)$, este valor indica una correlación positiva entre cada uno de ellos; es decir, se produjo un adecuado consenso en el desempeño de evaluación de los consumidores. Los Análisis de Procrustes Generalizado (GPA) realizados por Fukuda et al. (2017) para zumos de manzana, mencionan que el coeficiente de correlación se encontró valores relativamente altos $(0,713$ y 0,577$)$; sin embargo, para el caso de vino obtuvieron un coeficiente de correlación Rc $=0,304$, evidenciando que hay mucho menos acuerdo entre los evaluadores para productos más complejos, como en el caso del vino, que para productos menos complejos como en el caso de zumo de manzana.

En la Figura 1(a) se observa la agrupación realizada por los panelistas de acuerdo a la similitud de los atributos que estos perciben, el primer grupo está conformado por las muestras $G$ y $\mathrm{H}$, el segundo grupo por las muestras $\mathrm{A}$ y $\mathrm{B}$, el tercer grupo por las muestras $C$ y $D$ y finalmente un cuarto grupo conformado por las muestras $E$ y F. Por lo que los consumidores no perciben la 
variación del porcentaje de coagulante (lactato de calcio) sobre su percepción sensorial de estas muestras, independientemente del \% de lactato utilizado ( $305 \%$ ), los consumidores los describen y agrupan como muestras similares. Zhu et al. (2016) argumentan que utilizando cloruro de magnesio ( $\mathrm{MgCl} 2$ ) como coagulante modifica sus características del tofu, al aumentar la concentración de coagulante conduce a la formación de un gel de proteína más gruesa y también puede afectar a nivel de la microestructura del tofu.
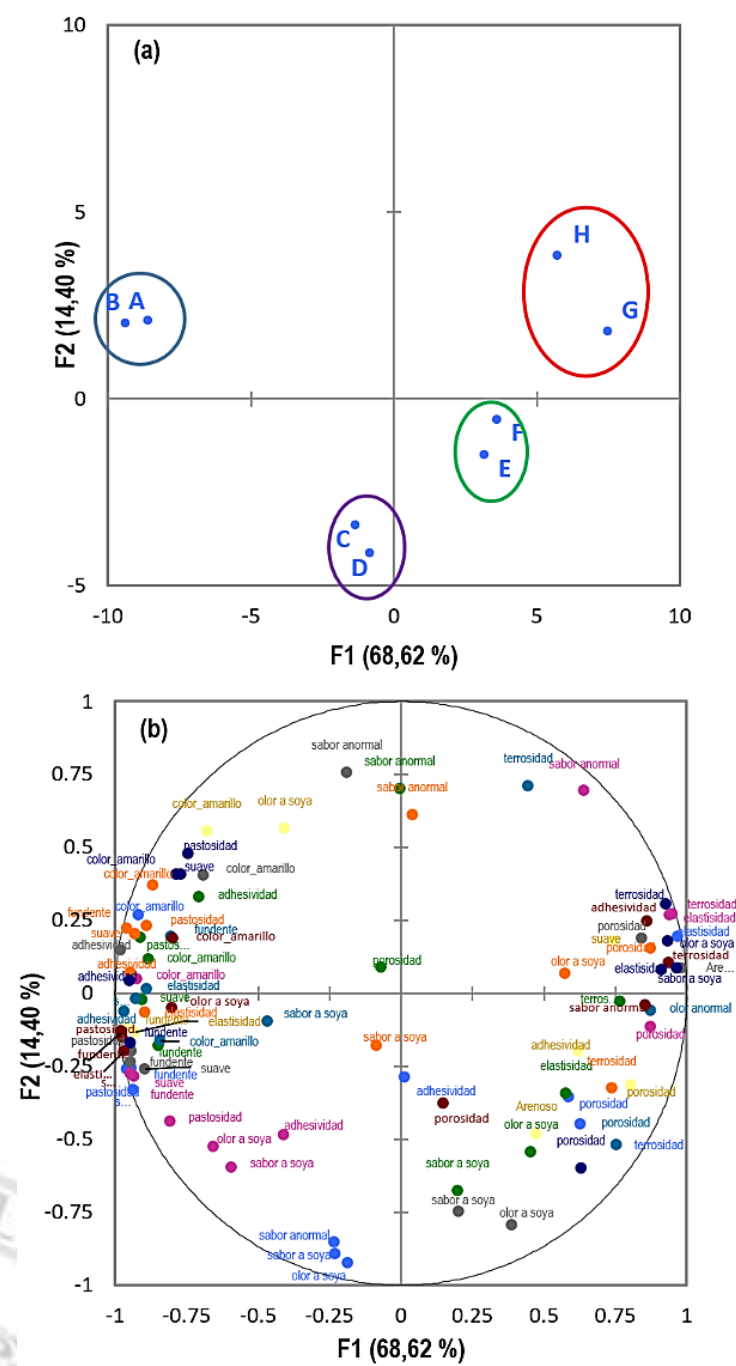

Figura 1. Análisis Procrustes Generalizado (a) agrupación y (b) términos de las muestras evaluadas.

En la Figura 1 (b) muestra el espacio sensorial de cada uno de los atributos descritos por los consumidores. Relacionando las Figuras 1 y 2, las muestras $\mathrm{H}$ y $\mathrm{G}$ se caracterizan por ser suaves, porosas, adhesivas, terrosas, elásticas, olor a y olor a soya. Las muestras $C$ y $D$ se caracterizan por presentar características como sabor a soya, olor a soya, ser adhesivas y porosas. Mientras que las muestras $\mathrm{E}$ y $\mathrm{F}$ se caracterizan por ser adhesivas, porosas y elástica soya, arenosas y sabor a soya. Las muestras A y B se caracterizan tener un color amarillo, pastosas, adhesivas, elásticas, fundentes, suaves.

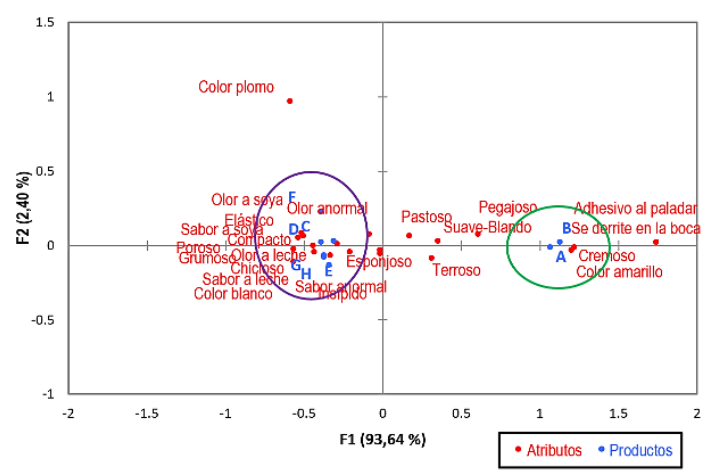

Figura 2. Mapa sensorial de análisis de correspondencia del método CATA.

\section{Resultados CATA}

De los 23 atributos considerados en la prueba de CATA los atributos olor anormal, esponjoso, sabor anormal, pastoso e insípido no presentaron diferencias significativas ( $p$-valor $>0,05$ ) entre las muestras según la prueba $Q$ de Cochran. El resto de los atributos presentaron diferencias significativas, los resultados son mostrados en la Tabla 2. Se encontró una relación altamente significativa entre el tipo de producto y el atributo según la prueba Chi-cuadrada ( $\mathrm{p}$-valor $<0,001$ ).

Del Prabhakaran et al. (2006) mencionan que el tipo de coagulante y el método de procesamiento, producen diferentes características de textura en el tofu como: suave, firme, gomoso, masticable. Del mismo modo, en las investigaciones realizadas por Cheng et al. (2005); Sun \& Breene, (1991) reportan que el tipo de coagulante y la concentración de este, afecta significativamente el rendimiento y las propiedades viscoelásticas del tofu. La temperatura de procesamiento y la relación agua/frijol también han influido en las cualidades texturales del tofu (Obatolu, 2008).

De la investigación realizada por Alamanou et al. (1996), al trabajar con aislado proteico de tarwi en la elaboración de salchicha, usándolo como aditivo, éste puede usarse hasta niveles de $2 \%$ ya que mejora los procesamientos y no afecta de manera significativa en los atributos como el color $y$ textura. A niveles superiores genera cambios significativos en el producto como el rendimiento y el sabor. La visualización de la tabla de contingencia para relacionar las muestras con los atributos se puede hacer a través de un análisis de correspondencias, cuyo mapa simétrico se muestra en la Figura 2. 
Tabla 2

Resultados de la prueba CATA

\begin{tabular}{|c|c|c|c|c|c|c|c|c|}
\hline Atributo & $A$ & $B$ & C & $D$ & $E$ & $\mathrm{~F}$ & G & $\mathrm{H}$ \\
\hline Suave ${ }^{* * *}$ & $31^{\mathrm{bc}}$ & $34^{c}$ & $23^{a b c}$ & $15^{a}$ & $17^{\mathrm{ab}}$ & $16^{\mathrm{ab}}$ & $12^{\mathrm{a}}$ & $17^{a b}$ \\
\hline C_Amarillo*** & $53^{b}$ & $50^{b}$ & $2^{\mathrm{a}}$ & $2^{\mathrm{a}}$ & $3^{a}$ & $1^{\mathrm{a}}$ & $3^{a}$ & $2^{\mathrm{a}}$ \\
\hline Terroso*** & $29^{b}$ & $29^{b}$ & $11^{\mathrm{a}}$ & $15^{\mathrm{ab}}$ & $24^{a b}$ & $14^{\mathrm{ab}}$ & $17^{\mathrm{ab}}$ & $12^{\mathrm{a}}$ \\
\hline Adhesivo*** & $47^{b}$ & $47^{b}$ & $0^{\mathrm{a}}$ & $0^{\mathrm{a}}$ & $0^{\mathrm{a}}$ & $0^{\mathrm{a}}$ & $0^{\mathrm{a}}$ & $0^{\mathrm{a}}$ \\
\hline Cremoso*** & $34^{b}$ & $34^{b}$ & $5^{a}$ & $4^{a}$ & $4^{a}$ & $2^{\mathrm{a}}$ & $2^{\mathrm{a}}$ & $3^{a}$ \\
\hline Fundente ${ }^{\star * *}$ & $31^{b}$ & $31^{b}$ & $0^{\mathrm{a}}$ & $0^{\mathrm{a}}$ & $0^{\mathrm{a}}$ & $0^{\mathrm{a}}$ & $0^{a}$ & $0^{\mathrm{a}}$ \\
\hline C_Blanco*** & $0^{\mathrm{a}}$ & $1^{\mathrm{a}}$ & $35^{b}$ & $36^{b}$ & $35^{b}$ & $27^{b}$ & $38^{b}$ & $38^{b}$ \\
\hline Sabor soya*** & $2^{\mathrm{a}}$ & $2^{\mathrm{a}}$ & $18^{b}$ & $23^{b}$ & $22^{b}$ & $24^{b}$ & $16^{\mathrm{ab}}$ & $20^{b}$ \\
\hline Compacto** & $9^{a}$ & $g^{a}$ & $22^{\mathrm{a}}$ & $18^{a}$ & $21^{a}$ & $23^{a}$ & $23^{a}$ & $23 a$ \\
\hline Olor leche $e^{\star \star *}$ & $13^{\mathrm{ab}}$ & $5^{\mathrm{a}}$ & $17^{\mathrm{ab}}$ & $18^{\mathrm{ab}}$ & $14^{\mathrm{ab}}$ & $12^{\mathrm{ab}}$ & $11^{\mathrm{ab}}$ & $20^{\mathrm{b}}$ \\
\hline Olor soya*** & $2^{\mathrm{ab}}$ & $1^{a}$ & $22^{c}$ & $23^{c}$ & $16^{b c}$ & $20^{c}$ & $20^{c}$ & $14^{\mathrm{abc}}$ \\
\hline Elástico*** & $2^{\mathrm{ab}}$ & $1^{\mathrm{a}}$ & $17^{\mathrm{bc}}$ & $30^{\text {cd }}$ & $34 d$ & $34^{d}$ & $23^{c d}$ & $24^{c d}$ \\
\hline Sabor leche $e^{\star * *}$ & $7^{\mathrm{ab}}$ & $4^{a}$ & $16^{a b c}$ & $21^{c}$ & $12^{\mathrm{abc}}$ & $11^{a b c}$ & $16^{\mathrm{abc}}$ & $17^{b c}$ \\
\hline Grumoso*** & $1^{a}$ & $0^{\mathrm{a}}$ & $24^{b}$ & $29 \mathrm{~b}$ & $30^{\mathrm{b}}$ & $28^{b}$ & $26^{b}$ & $29 \mathrm{~b}$ \\
\hline Chicloso*** & $6^{a b}$ & $5^{\mathrm{a}}$ & $22^{\mathrm{bc}}$ & $29 c$ & $28 \mathrm{c}$ & $26^{c}$ & $27 c$ & $29 c$ \\
\hline Poroso*** & $7^{a}$ & $6^{a}$ & $32^{b}$ & $37^{b}$ & $32^{b}$ & $37^{b}$ & $41 b$ & $38^{b}$ \\
\hline Pegajoso*** & $27^{b}$ & $32^{b}$ & $10^{\mathrm{a}}$ & $10^{\mathrm{a}}$ & $7^{\mathrm{a}}$ & $11^{\mathrm{a}}$ & $8^{a}$ & $11^{\mathrm{a}}$ \\
\hline C_Gris ${ }^{* * *}$ & $0^{a}$ & $0^{\mathrm{a}}$ & $4 a b$ & $4^{a b}$ & $0^{a}$ & $10^{b}$ & $2^{a}$ & $2^{\mathrm{a}}$ \\
\hline Olor anormal ${ }^{*}$ & $30^{\mathrm{a}}$ & $24^{a}$ & $35^{a}$ & $24^{a}$ & $27^{a}$ & $35^{a}$ & $34^{a}$ & $37^{a}$ \\
\hline Esponjoso* & $g^{a}$ & $11^{a}$ & $13^{\mathrm{a}}$ & $11^{a}$ & $13^{\mathrm{a}}$ & $9^{a}$ & $g^{a}$ & $9^{a}$ \\
\hline Sabor anormal* ${ }^{*}$ & $24^{a}$ & $17^{a}$ & $18^{a}$ & $15^{a}$ & $26^{a}$ & $22^{a}$ & $28^{a}$ & $18^{a}$ \\
\hline Pastoso* & $47^{a}$ & $5^{a}$ & $47^{a}$ & $35^{\mathrm{a}}$ & $32^{\mathrm{a}}$ & $39 a$ & $34^{a}$ & $37^{a}$ \\
\hline Insípido* & $43^{a}$ & $45^{a}$ & $41^{\mathrm{a}}$ & $47^{a}$ & $43^{a}$ & $52^{a}$ & $43^{a}$ & $45^{a}$ \\
\hline
\end{tabular}

Los asteriscos indican el nivel de significancia según prueba de Cochran, $\left({ }^{* * *}\right)$ significativo al 0.001 , $\left({ }^{* *}\right)$ significativo al $0.01, y\left({ }^{*}\right)$ significativo al 0,05 . Las letras que están como exponente hacen referencias a que dos atributos que comparten las mismas letras no difieren significativamente. Dos atributos que no tienen letras en común difieren significativamente.

Se infiere que los productos A y B se caracterizan por ser cremoso, adhesivo, fundente y de color amarillo, mientras que el tofu con mezcla soyatarwi se caracterizó por ser más compacto, elástico, de color blanco, con sabor a soya. Las proteínas de soya tienen la capacidad de mejorar y estabilizar la emulsión de grasa, mejorar la viscosidad, impartir textura tras la gelificación después de la cocción y mejorar la retención de humedad y rendimientos generales (Alamanou et al., 1996).

Las muestras A y $B$, hechas a base de semillas de tarwi, presentan características muy diferentes a las muestras hechas con soya, una de las características que más se resalta es que estas muestras son más suaves y no presentan la forma compacta como las demás muestras. Yasin et al. (2019) mencionan que las investigaciones realizadas a las propiedades fisicoquímicas y sensoriales del tofu a partir de ocho variedades de soya tenían una textura firme y compacta y esto fue logrado por el alto contenido de proteína que contiene la soya. La proteína de la soya es el componente principal del tofu, esta proteína es la glicina (11S) y $\beta$-conglicinina (7S). Estos dos componentes proteicos conforman más del $70 \%$ de la proteína total presente en el tofu (Wang et al., 2020). Diversas investigaciones han demostrado que la relación $11 \mathrm{~S} / 7 \mathrm{~S}$ está relacionada con la dureza del tofu (Meng et al. 2016; Niu et al. 2018).

\section{Comparación de resultados del perfil libre elección y CATA}

Ares et al. (2010) mencionan que al trabajar los métodos CATA y mapeo proyectivo con descriptores, la proximidad de los resultados obtenidos proporciona información. Del mismo modo, Liu et al. (2018) reportan que, al trabajar con diferentes metodologías sensoriales descriptivas rápidas, entre ellas el método de perfil de libre elección, estas muestran resultados similares, además que los evaluadores la consideran como una metodología fácil para evaluar los productos.

Como se muestra en la Figura 3, el Análisis Multifactorial Jerárquico (HMFA) comparación de los dos métodos (perfil de libre elección y CATA), tenían distancias similares, al utilizar cualquiera de los dos métodos, se muestran resultados similares. Es decir, tanto en el perfil libre y el CATA, tiene similitud entre sí, para confirmar esta similitud se utilizó el coeficiente con el cual fue de 0,94 , confirmando la similitud entre estos dos métodos. 


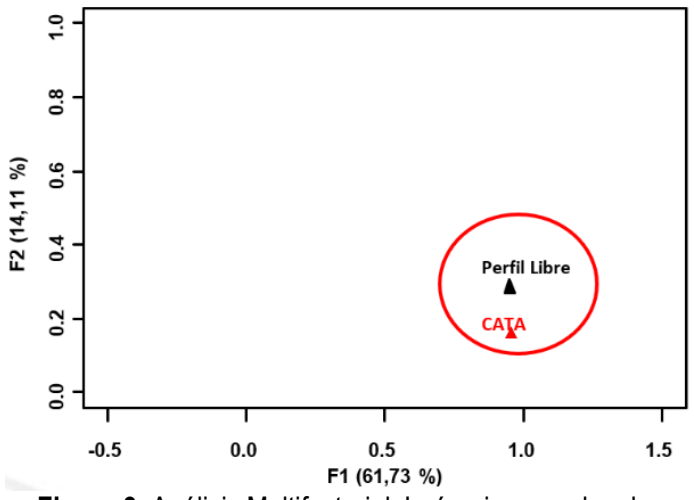

Figura 3. Análisis Multifactorial Jerárquico para los dos métodos descriptivos.

\section{Conclusiones}

La evaluación de los métodos descriptivos (Perfil de libre elección y CATA) por los consumidores, permite describir las características del tofu. Con la aplicación de estos métodos se pudo lograr identificar las características atribuidas al tofu. Respecto, al perfil de libre elección, los evaluadores pudieron describir de forma libre los atributos percibidos, comprendiendo éstos principalmente en color, sabor y textura. Del mismo modo, el método CATA, los evaluadores pudieron distinguir las diferentes muestras marcando atributos diferentes. En base a los resultados, ambos métodos pueden ser utilizados para la descripción de las características del tofu y otros productos.

\section{Referencias bibliográficas}

Alamanou, S., Bloukas, J. G., Paneras, E. D., \& Doxastakis, G. (1996). Influence of protein isolate from lupin seeds (Lupinus albus ssp. Graecus) on processing and quality characteristics of frankfurters. Meat science, 42(1), 79-93.

Ares, G., Deliza, R. Barreiro, C., Giménez, A., \& Gámbaro, A. (2010). Comparison of two sensory profiling techniques based on consumer perception. Food quality and preference, 21(4), 417426.

Bauer, M., Mitchell, S., Elliott, N., \& Roberts, J. (2020). Rapid sensory profiling of tennis rackets. In Multidisciplinary Digital Publishing Institute Proceedings (Vol. 49, No. 1, p. 123)

Cheng, Y., Shimizu, N., \& Kimura, T. (2005). The viscoelastic properties of soybean curd (tofu) as affected by soymilk concentration and type of coagulant. International journal of food science \& technology, 40(4), 385-390.

Elmore, J. R., \& Heymann, H. (1999). Perceptual maps of photographs of carbonated beverages created by traditional and free-choice profiling. Food quality and preference, 10(3), 219227

Esmerino, E. A., Tavares Filho, E. R.. Carr, B. T., Ferraz, J. P., Silva H. L., Pinto, L. P. F., Freitas, M. Q., Cruz, A. G \& Bolini, H. M. (2017). Consumer-based product characterization using Pivot Profile, Projective Mapping and Check-all-that-apply (CATA): A comparative case with Greek yogurt samples. Food Research International, 99, 375-384.

Fukuda, M., Kobayashi, M., \& Honda, Y. (2017). Functional Components and Health Benefits of Fermented Soymilk. In Soft Chemistry and Food Fermentation (pp. 145-178). Academic Press.
Kharlamova, A., Nicolai, T. \& Chassenieux, C. (2018). Mixtures of sodium caseinate and whey protein aggregates: Viscosity and acid-or salt-induced gelation. International Dairy Journal, 86, 110-119

Li, C., Rui, X., Zhang, Y., Cai, F., Chen, X., \& Jiang, M. (2017). Production of tofu by lactic acid bacteria isolated from naturally fermented soy whey and evaluation of its quality. LWT-Food Science and Technology, 82, 227-234.

Liu, J., Bredie, W. L., Sherman, E., Harbertson, J. F., \& Heymann, H. (2018). Comparison of rapid descriptive sensory methodologies: Free-choice profiling, flash profile and modified flash profile. Food Research International, 106, 892-900.

Meng, S., Chang, S., Gillen, A. M., \& Zhang, Y. (2016). Protein and quality analyses of accessions from the USDA soybean germplasm collection for tofu production. Food chemistry, 213, 31-39.

Murugkar, D. A. (2015). Effect of different process parameters on the quality of soymilk and tofu from sprouted soybean. Journal of food science and technology, 52(5), 2886-2893.

Narain, C., Paterson, A., \& Reid, E. (2004). Free choice and conventional profiling of commercial black filter coffees to explore consumer perceptions of character. Food Quality and Preference, 15(1), 31-41.

Nascimento, R. Q., Tavares, P. P. L. G., Meireles, S., Anjos, E. A. D., Andrade, R. B. D., et al. (2020). Study on the sensory acceptance and check all that apply of mixed juices in distinct Brazilian regions. Food Science and Technology, 40, 708-717.

Nikolić, Z., Petrović, G., Panković, D., Ignjatov, M., Marinković, D. Stojanović, M., \& Đorđević, V. (2017). Threshold level and traceability of roundup ready $($ ) soybeans in tofu production. Food technology and biotechnology, 55, 439-444.

Niu, H., Xia, X., Wang, C., Kong, B., \& Liu, Q. (2018). Thermal stability and gel quality of myofibrillar protein as affected by soy protein isolates subjected to an acidic $\mathrm{pH}$ and mild heating. Food chemistry, 242, 188-195

Obatolu, V. A. (2008). Effect of different coagulants on yield and quality of tofu from soymilk. European Food Research and Technology, 226(3), 467-472.

Pearson, W. Schmidtke, L, Francis, I. L. \& Blackman, J. W. (2020). An investigation of the PivotC Profile sensory analysis method using wine experts: Comparison with descriptive analysis and results from two expert panels. Food Quality and Preference, 83, 103858.

Pelonnier-Magimel, E., Windholtz, S., Pomarède, I. M. \& Barbe, J. C. (2020). Sensory characterisation of wines without added sulfites via specific and adapted sensory profile. OENO One, 54(4), 671-685

Pizzutti, I. R., de Kok, A., Zanella, R., Adaime, M. B., Hiemstra, M., Wickert, C., \& Prestes, O. D. (2007). Method validation for the analysis of 169 pesticides in soya grain, without clean up, by liquid chromatography-tandem mass spectrometry using positive and negative electrospray ionization. Journal of Chromatography A, 1142(2), 123-136.

Prabhakaran, M. P.., Perera, C. O. \& Valiyaveettil, S. (2006). Effect of different coagulants on the isoflavone levels and physical properties of prepared firm tofu. Food Chemistry, 99(3), 492499

Sun, N., \& Breene, W. M. (1991). Calcium sulfate concentration influence on yield and quality of tofu from five soybean varieties. Journal of Food Science, 56(6), 1604-1607.

Tarancón, P., Tárrega, A., Aleza, P., \& Besada, C. (2020). Consumer Description by Check-All-That-Apply Questions (CATA) of the Sensory Profiles of Commercial and New Mandarins. Identification of Preference Patterns and Drivers of Liking. Foods, 9(4), 468

Van de Noort, M. (2017). Lupin: an important protein and nutrient source. In Sustainable protein sources (pp. 165-183). Academic Press.

Vit, P., Pedro, S. R., Vergara, C., \& Deliza, R. (2017). Ecuadorian honey types described by kichwa community in rio chico, Pastaza province, Ecuador using free-choice profiling. Revista Brasileira de Farmacognosia, 27(3), 384-387.

Wang, F., Meng, J., Sun, L., Weng, Z., Fang, Y., Tang, X., ... \& Shen, $X$. (2020). Study on the tofu quality evaluation method and the 
establishment of a model for suitable soybean varieties for Chinese traditional tofu processing. LWT, 117, 108441.

Yasin, U. A., Horo, J. T., \& Gebre, B. A. (2019). Physicochemical and sensory properties of tofu prepared from eight popular soybean [Glycine max (L.) Merrill] varieties in Ethiopia. Scientific African, 6, e00179.
Zhang, Q., \& Qin, W. (2019). Tofu and Soy Products: The Effect of Structure on Their Physicochemical Properties.

Zhu, Q., Wu, F., Saito, M., Tatsumi, E., \& Yin, L. (2016). Effect of magnesium salt concentration in water-in-oil emulsions on the physical properties and microstructure of tofu. Food Chemistry, 201, 197-204.

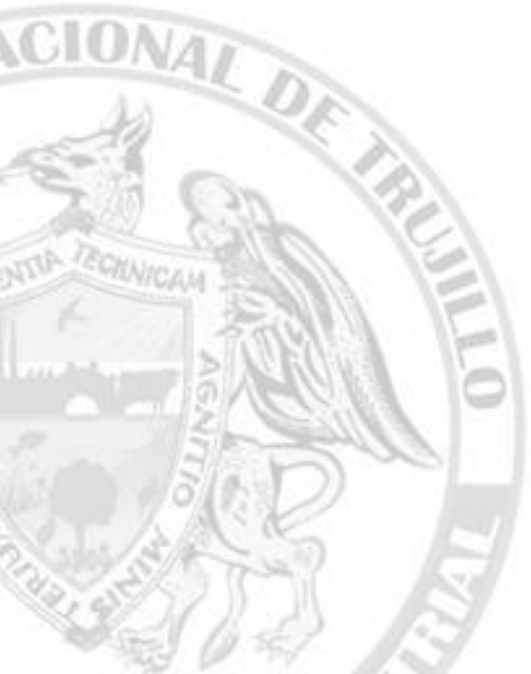

